\title{
The Neurospora circadian clock-controlled gene, $c c g-2$, is allelic to eas and encodes a fungal hydrophobin required for formation of the conidial rodlet layer
}

\author{
Deborah Bell-Pedersen, Jay C. Dunlap, ${ }^{1}$ and Jennifer J. Loros ${ }^{1}$ \\ Department of Biochemistry, Dartmouth Medical School, Hanover, New Hampshire 03755 USA
}

\begin{abstract}
The Neurospora crassa clock-controlled gene (ccg-2) is transcriptionally activated by the circadian clock in a time-of-day-specific manner. Transcript and sequence analyses of $c c g-2$ reveal that the predicted $\mathrm{Ccg}-2$ polypeptide bears significant similarity to a class of low-molecular-weight, cysteine-rich, hydrophobic proteins (hydrophobins), first identified in Schizophyllum, and including the product of the developmentally regulated Aspergillus gene, rodletless, required for spore surface rodlets. Allelism between ccg-2 and easily wettable (eas) (one of the first developmental genetic loci identified in Neurospora) was predicted on the basis of this similarity, their close genetic linkage, and previous findings demonstrating that eas mutants lack rodlets. In this study allelism is confirmed experimentally by showing that (1) transformation of an eas mutant strain with ccg-2 DNA results in phenotypic complementation, including restoration of surface rodlets, (2) inactivation of the ccg-2 gene, by RIP, results in an eas phenotype including loss of rodlet fascicles, and (3) the original eas strain has dramatically reduced levels of $c c g-2$ mRNA. Thus, the clock-controlled $c c g-2$ gene encodes an integral component of fungal asexual spores important for spore dispersal. The dramatic reduction of $c c g-2$ expression in the eas mutant has no apparent effect on the normal operation of the circadian clock, confirming that there is no feedback of this clock output on the oscillator itself. These data, in conjunction with the previous observation that $c c g-2$ is light induced, serve to focus attention on the dual interacting role of light and the circadian clock in the regulation of fungal spore development.
\end{abstract}

[Key Words: Clock-controlled gene; ccg-2; easily wettable; hydrophobins; rodlets; circadian clock]

Received August 13, 1992; revised version accepted September 15, 1992.

Circadian rhythms are biological rhythms that are present in most, if not all, eukaryotes (Dunlap 1990). Daily fluctuations in cellular and organismal activities are controlled by an endogenous oscillator, commonly referred to as the biological clock. The clock exerts its effects on a wide variety of processes ranging from asexual spore formation in the ascomycete Neurospora crassa and the control of cell division in the marine protist Gonyaulax to photosynthesis in plants and cognitive functions in people (for review, see Edmunds 1988). However, at all phylogenetic levels, clocks appear to be assembled at the level of intracellular regulation and not intercellular communication. Thus, simple eukaryotes provide appropriate and powerful systems for molecular genetic analysis of this phenomenon.

Of the eukaryotes known to possess a well-defined circadian clock, the filamentous fungus $N$. crassa provides an ideal model system for clock analysis (for review, see Feldman and Dunlap 1983; Dunlap 1990; Lakin-Thomas

${ }^{1}$ Corresponding authors. et al. 1990|. Advantages include an easily measurable circadian rhythm in developmental potential (rhythmic formation of aerial hyphae and asexual spores, called conidia), the ability to manipulate and synchronize the clock with light, temperature, or drugs, and the availability of strains with genetically altered clocks. With regard to clock mutants, one locus, frequency $($ fr $q)$, is of particular interest because independently isolated mutants mapping to the frq locus are able to alter one or more clock parameters, including period length and temperature compensation (for review, see Dunlap 1990; Lakin-Thomas et al. 1990). For these reasons, we have chosen $N$. crassa as a model organism for investigating the pathways by which the clock functions to relay time information throughout the cell.

One approach to understanding the mechanisms by which the clock controls cellular metabolism is through the identification and analysis of genes or proteins that are expressed in a circadianly regulated fashion, in the same manner in which one might identify and study developmentally regulated genes. In a systematic comparison of mRNAs isolated from synchronous $N$. crassa 
cultures representing subjective early morning [circadian time $1(\mathrm{CT} 1)]$ and early evening (CT13), Loros et al. (1989) isolated two morning-specific circadianly regulated clock-controlled genes (ccg-1 and ccg-2). (Circadian time is a formalism whereby biological time in strains or organisms with different endogenous period lengths is normalized to 24 circadian hours per cycle. By convention, CTO represents subjective dawn, and CT12 represents subjective dusk.) The circadian nature of the regulation of these genes was discerned by examining the pattern of their RNA accumulation in both $\mathrm{frq}^{+}(21.5 \mathrm{hr}$ period length) and a long period mutant strain, $f r q^{7}(29 \mathrm{hr}$ period length), where transcripts arising from the clockcontrolled genes cycled with period lengths reflecting the genotype (i.e., 21.5 or $29 \mathrm{hr}$ ). Both genes are abundantly expressed at CT0-CT1, display an $\sim 10$ - to 15 -fold decrease in expression at the opposite phase of the cycle (CT12-CT13), and both appear to be light inducible (Dunlap 1990; G. Arpaia, J. Loros, J.C. Dunlap, and G. Macino, in prep.). Nuclear run-on experiments performed on both $c c g-1$ and $c c g-2$ indicate that rhythmic expression results from clock control of transcription (Loros and Dunlap 1991). This regulation suggests that clock control of gene expression for ccg-1 and ccg-2 is mediated by cis-acting regulatory elements located within or near these genes.

The biological function of clock-controlled genes is of considerable interest. The study of rhythmic genes will ultimately provide information about the mechanism by which time information is transmitted within the cell. Additionally, determination of the identity of the ccgs will provide insight into the rationale for clock regulation of development and the means by which this regulation is effected. In this study we describe our analysis of the N. crassa ccg-2 gene. Molecular studies of $c c g-2$ reveal that the gene product is involved in the formation of the thin, proteinaceous, hydrophobic rodlet layer present on conidial surfaces (Hawker and Madelin 1976; Beever and Dempsey 1978; Beever et al. 1979; Dempsey and Beever 1979). Complementation of an N. crassa rodlet-deficient mutant [easily wettable (eas)]; Selitrennikoff 1976) with ccg-2 DNA, and generation of the eas phenotype by inactivation of the $c c g$-2 gene provides evidence that $c c g-2$ and eas are allelic. These data demonstrate a role for circadian regulation in the development of filamentous fungi and their selective adaptation to the environment in which they grow.

\section{Results}

ccg-2 encodes a clock-regulated hydrophobin

Circadian control of ccg- 2 mRNA abundance and mapping of the ccg-2 transcription unit. Clock control of $c c g-2$ expression is observed in RNA isolated at different times of the day from mycelia grown in constant darkness (Loros et al. 1989). The ccg-2 message (776 nucleotides) is present at minimal levels in the evening (CT12) and increases in abundance $\sim 10$ - to 15-fold in the morning (CT0) (Fig. 1A and Loros et al. 1989).
The gene encoding $c c g-2$ was first isolated from a $\lambda \mathrm{J} 1$ genomic library (clone 7C1; Loros et al. 1989). An $\sim 6$-kb $X b a I$ fragment containing the $c c g-2$ transcriptional unit was subcloned from $7 \mathrm{Cl}$ and inserted into pBluescript + (pBSK + , Stratagene). The resulting plasmid, pS7C1, was used as a template to further define the $c c g$-2 transcription unit. Rhythmic RNA was probed with various length restriction fragments generated from $\mathrm{pS7Cl}$ (Fig. 1B). Circadianly regulated $c c g-2$ mRNA was detected using a $1.9-\mathrm{kb} K p n I-X b a I$ DNA fragment as a probe but was not detected using sequences upstream of the KpnI site or downstream of the $X b a I$ site (data not shown). This indicated that the $c c g-2$ transcriptional unit was contained within the 1.9-kb KpnI-XbaI fragment.

Sequence and transcriptional analysis of ccg-2. The 1.9-kb KpnI-XbaI genomic fragment and several ccg-2 cDNA clones were sequenced (Fig. 2). The ends of the transcript were mapped by primer extension and $\mathrm{Sl}$ nuclease protection assays, with the $5^{\prime}$ end indicated as nucleotide 1 and the $3^{\prime}$ end as nucleotide 869 (the last nucleotide of the $c c g-2$ sequence; Fig. $2 B \mid$. The poly(A) addition site was corroborated by sequencing the 3 ' end of $c c g-2$ cDNAs. Sequence comparisons of cDNA to genomic DNA revealed an intron of 94 nucleotides, which when spliced yields a mature message of 776 nucleotides. The splice site consensus sequences are typical of $N$. crassa intervening sequences (Gurr et al. 1987) and are underscored in Figure 2B. A putative TATA box (at -39 ) and a putative CAAT box (at -254) were identified and are indicated in Figure 2, A and B.

The $c c g-2$ gene is predicted to encode a polypeptide of 108 amino acids (Fig. 2B), with an estimated $M_{\mathrm{r}}$ of 11,000 . The codon bias of the $c c g-2$ open reading frame (ORF) is consistent with genes that are highly expressed in N. crassa (Gurr et al. 1987). Overall, hydrophobic amino acids predominate in Ccg-2 (45\% hydrophobic residues: A, F, I, L, M, P, V), with the protein containing a strong hydrophobic amino terminus [characteristic of a signal sequence (Boyd and Beckwith 1990)] and a central hydrophobic domain as shown in Figure 3. In addition, the ORF specifies an unusually high number of cysteine residues (8). The polypeptide contains potential sites of phosphorylation by casein kinase 2 (amino acids SIDD following residue 36 ) and protein kinase $\mathrm{C}$ (amino acids SVK following residue 83), and three overlapping potential myristylation sites (amino acids GCVVGV at residue 70, GVIGSQ at residue 74, and GSQCGA at residue 77) occur near the end of the ORF in a relatively more conserved region of the protein (see below; Fig. 3A).

Comparison of the Ccg-2 amino acid sequence with protein data bases (SWISS-PROT and NBRF-PIR protein) revealed that $\mathrm{Ccg}-2$ protein shares significant sequence similarity to several low-molecular-weight, cysteinerich fungal proteins involved in cell wall hydrophobicity (called hydrophobins). Three hydrophobin genes, Sc1, Sc3, and Sc4 from Schizophyllum commune are abundantly expressed in fruiting dikaryons /as well as in nonfruiting monokaryons and dikaryons for Sc3) (Schuren and Wessels 1990). Each protein shares extensive se- 
Figure 1. Clock regulation and subcloning of $c c g-2$. (A) Rhythmic expression of $c c g$-2. Mycelia from control strain $b d A$ was grown in liquid culture (Materials and methods) in constant dark and harvested after $32,36,18,22,25,8$, and $12 \mathrm{hr}$ in the dark representing $\sim \mathrm{CT0}, \mathrm{CT} 4, \mathrm{CT} 8$, CT12, CT16, CT21, and CT1, respectively. In wild-type clock strains this represents one full circadian cycle. Total RNA was extracted, and $10 \mu \mathrm{g}$ of RNA was loaded on a denaturing gel. The RNA was transferred to a nylon membrane and hybridized to a radiolabeled $c c g-2 \mathrm{KpnI}(\mathrm{K})-$ $X b a \mathrm{I}(\mathrm{X})$ DNA probe (shown in B). Following autoradiography, the relative amount of $c c g-2$ message was quantitated by densitometry and plotted as relative band intensity vs. circadian time. Constant loading was verified by examination of the amount of rRNA in each lane (data not shown), levels of which have been shown to remain constant over time under these growth conditions (Loros et al. 1989). Clock-controlled morning-enhanced expression of $c c g-2$ mRNA is evident from the observed 14-fold increase of $c c g$ - 2 message at CT0 and CT1, as compared with the opposite phase of the cycle at CT12. A more detailed description of the clock-regulated expression of $c c g-2$ mRNA can be found in Loros et al. (1989). (B) Localization of the $c c g-2$ gene. Genomic clone $7 \mathrm{Cl}$ (Loros et al. 1989) was subcloned as a 6-kb XbaI fragment into $\mathrm{pBSK}^{+}$yielding plasmid pS7Cl. This 6-kb XbaI fragment (top) hybridizes to the 776-nucleotide morning-specific $c c g-2$ RNA. The restriction fragments shown below were used to locate the $c c g-2$ transcription unit. Plus signs $(+)$ indicate the ability of the fragment to hybridize to $c c g-2$ mRNA, whereas the minus sign $(-)$ designates a lack of detection of ccg-2 message.

quence homology, with 8 conserved cysteines and a hydrophobic amino terminus (likely to include a signal sequence), and an internal hydrophobic domain, similar to Ccg-2. Recently, sequence analysis of the developmentally regulated rodletless (rodA) locus from Aspergillus nidulans revealed the same arrangement of 8 cysteine residues and hydrophobic regions observed in the $S$. commune genes (Stringer et al. 1991), as observed for Ccg-2. The Ccg-2, RodA, and Sc3 gene products are shown aligned in Figure 3A. Overall, the three sequences show $16 \%$ identity, with $52 \%$ conserved amino acid changes. Two-way pairing shows even greater similarity, and the positions of the $c c g-2$ intron and the 3 '-most $\operatorname{rod} A$ and $S c 3$ introns are similar, suggesting that all three gene products (as well as $\mathrm{Scl}$ and Sc4) originated from a common ancestor. The hydrophobic amino termini of these related proteins indicates that they are secreted, as has been demonstrated for the Scl, Sc3, and Sc4 hydrophobins (Wessels et al. 1991).

Direct inactivation of the rodA locus results in strains that lack the parallel bundles of rodlets typically found on the outer surface of the conidia (Stringer et al. 1991), a characteristic noted to be reminiscent of the previously identified $N$. crassa mutant eas allele UCLA191;
Selitrennikoff 1976) (Beever and Dempsey 1978). Additionally, the asexual spores of both eas and $\operatorname{rod} A$ have a darkened, wetted appearance, and are not readily dispersed in air.

\section{ccg-2 is allelic to the developmental locus eas}

The $N$. crassa eas mutation has been genetically mapped to linkage group II (Selitrennikoff 1976), very close to the location of ccg-2 as defined by restriction fragment length polymorphism (RFLP) mapping (Loros et al. 1989). Together, the map location of eas, the striking homology of Ccg-2 to RodA protein, and the similarity of developmental phenotype between eas and rodA suggested that $c c g-2$ might be allelic to eas. This was supported further by subsequent experiments (see below).

DNA-encoding ccg- 2 rescues the eas mutant phenotype. We first tested the ability of $c c g-2$ DNA to rescue the eas mutant phenotype. A ccg-2 construct (pLW1), containing a 3-kb EcoRI-XbaI ccg-2 genomic DNA fragment (with $\sim 1.7 \mathrm{~kb}$ of upstream sequences) was linearized at the unique $\mathrm{XbaI}$ site, and the Aspergillus $\operatorname{trp} C$ promoter-driven hygromycin phosphotransferase gene 
A

DNA

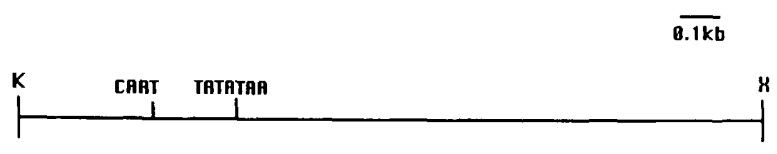

MRNA

$\mathbf{5}^{\prime}$

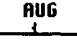

3
B

$-625$

$-545$

$-465$

$-385$

$-305$

$-225$

$-145$

$-65$

1

69
GGTACCCAATTCCGACAATCCCTTAGCTGGGTGAGATTTCACTGCTCAGTATCAATGGAAACATTGATGAGGCTCCATGT CTTTCCCGTTCCTGGGAGGAGCCACGTTCGTCATTGGCGCACTTCTAACCGTGTACACTAGTGTAGTTCAGAACAGTGCT GAATCATATGTGGTCGGACTTGGTGTTCCTGATGTCTGACATCTGGCAGCGAAAGGTGCTCTAGTGAAAGGATCGTCATT GCTCTTTCCAGATTCCGAGCCACGATGTCGGTTATCGTGGCCAGATCTCCAAACAAGCTAATGGAGGTTTGCGCCGCATA CTCGTTGCTGTTCCCCATTGGGAGCCCAGAGCATGATCTCGTTGTCACAATTCTTGATCGACAGCCCCAAGAGCCTGGGT CGATTGGGTCCGGATGAGTGCCAGGCCGGATGCCAGATCTGGAAATGACGAGTGTCGATGAATCAACAACACCAAGCCAC GGCGGATAGGAGGAAGGAGAAGCAGGAATACCGGAGAACTTGGCCAAGTTTGATGGACGAAGTCTTCAAACACAGCGTTG GGATTGAGGTCCAAAGTGTATATAAGACTCGTCTCTCGTCCACCTCAGAGCTTCTATTCTTCTC

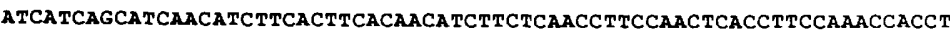
tCAAAACCAACTCCCAGCTTCTTTCAGCAAACCCCCAACCGCCAAa ATg CAg TTC ACC AGC
met gln phe thr ser GTC TTC ACC ATC CTC GCC ATT GCC ATG ACC GCC GCT GCG GCC CCG GCT GAG
val phe thr ile leu ala ile ala met thr ala ala ala ala pro ala glu GTT GTT CCC CGC GCC ACC ACC ATC GGC CCC AAC ACC TGC TCC ATC GAC GAC
val val pro arg ala thr thr 1 Te gly pro asn val pro arg ala thr thr lle gly pro asn thr cys ser ile asp asp TAC AAG CCT TAC TGC TGC CAG TCT ATG TCC GGC CCC GCC GGC TCC CCT GGT tyr lys pro tyr cys cys gin ser met ser gly pro ala gly ser pro gly CTC CTC AaC CTC ATC CCC GTC GAC CTC AGC GCC TCG CTC GGC TGC GTT GTC leu leu asn leu tle pro val asp leu ser ala ser leu gly cys val val GT GTC ATC GGC TCC CAA TGT GGT GCC AGC GTC AAG TGC TGC AAG GAC GAT gly val ile gly ser gln cys gly ala ser val lys cys cys lys asp asp

ACC AAC gtaagtttttcctcettctccctccacactacagcgegctctcttcagaaacagt val thr asn

49 cccettaataacettcgcttttgtgccgacgaaaaag ACC GGC AAC TCC TTC CTC ATC thr gly asn ser phe leu ile

8 ATC AAC GCT GCC AAC TGC GTT GCC taA GTgtttacgcggcaAcagcGcaAagtctaggC ile asn ala ala asn cys val ala stop

7 AatgCCTTGTTCTCAACGCTGCTGCCAGTCCAGCACCCCCCTTCTGCAGCAAGGAGCCCCCTTCTGCT

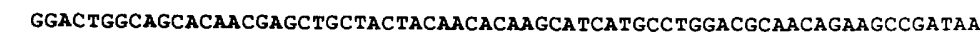

TCTTGGGGTTGGTTTTGGGGATGAAGGTGATGAGTTGATGGATTGGATCGATATCTTACAATGCGT

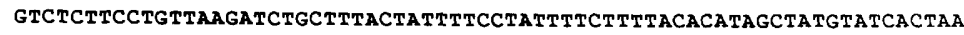
GGCCTGgTGATTAATACACTCTCTTAACCCT*
Figure 2. Transcriptional organization and sequence of $c c g$-2. $(A) c c g-2$ genomic DNA and RNA maps. Schematic representation of the 1.9kb KpnI (K)-XbaI (X) genomic ccg-2 DNA fragment and its corresponding transcript. The putative CAAT box at -254 and the TATA box at -39 are indicated on the DNA. The direction of transcription of $c c g-2$ mRNA is from left to right. The translational signals (AUG and UAA) are shown. The position of the intervening sequence is shown as being removed from the RNA. $|B|$ DNA sequence upstream of and including the $c c g-2$ transcription unit. The major start site of transcription $(+1)$ was determined by primer extension analysis. The poly(A) addition site $\left({ }^{*}\right)$ was established by S1 protection studies and verified from $c c g-2$ cDNA sequence analysis. Both the putative CAAT and TATA boxes are underscored. The single 94-nucleotide intron is in lowercase letters, with the consensus splice sites (Gurr et al. 1987) underscored. The protein sequence corresponding to the longest ORF, starting with the first AUG codon after the transcriptional start site, is given below the sequence. The EMBL data library accession number is $\mathrm{X} 67339$. (hph) (from pDH25; Cullen et al. 1987) was inserted. The resulting plasmid, pLW1H (Fig. 4A) was transformed into the easA mutant strain UCLA191 yielding transformants TDP160-180. Of these 20 hygromycin-resistant, randomly inserted $\mathrm{pLW} 1 \mathrm{H}$ transformants, 4 were found to produce wild-type conidia (TDP176-180) that were light orange in color and readily dispersed in air. Transformation with the plasmid vector alone did not rescue easA. Because transformation in $N$. crassa is typically ectopic, resulting from nonhomologous recombination of the circular vector into chromosomal DNA, the combined effects of interruption of plasmid-borne genes by the recombination event and chromosomal positioning effects on expression often result in a reduced frequency of expression of transformed genes (e.g., McClung et al. 1989). Thus, the observed rescue efficiency of $\sim 20 \%$ is consistent with allelism of $c c g-2$ and eas. In four separate experiments, transformation of eas strains with similar ccg- 2 constructs containing only the $1.9-\mathrm{kb} K p n I-X b a \mathrm{I}$ fragment (with 625 nucleotides of upstream sequences) was unable to rescue the eas phenotype (data not shown), possibly owing to the lack of requisite upstream cis-acting sequence elements in this construct (see Discussion).

To demonstrate that the wild-type conidial phenotype observed in the rescued transformants (TDP176-180) was the result of ectopic integration and subsequent expression of the introduced copy of $c c g-2$, Southern analysis was performed on one of the eas-rescued transformants (TDP176) (Fig. 4B). Genomic DNAs from control strain bdA, rescued transformant TDP176, and easA (lanes 1-3, respectively) were digested with KpnI or NdeI. Following transfer to a nylon membrane, the DNA was hybridized to a labeled ccg-2 DNA probe. For both the KpnI and NdeI digests, a DNA fragment of the expected size from the endogenous copy of $c c g-2$ was observed in all three strains (5.2 and $7.2 \mathrm{~kb}$, respectively). Consistent with ectopic integration of a single copy of $\mathrm{pLW} 1 \mathrm{H}$, an additional single $c$ cg-2-specific fragment was detected for both digests in the TDP176 rescued strain, which was absent in the control strains $13.5 \mathrm{~kb}$ for KpnI 
Figure 3. Comparison of the predicted polypeptides encoded by $c c g-2, S c 3$, and $\operatorname{rodA}$. (A) Amino acid homologies. The conceptual translation sequence of $\mathrm{Ccg}-2$ (108 amino acids) is shown aligned with the $S$. commune Sc3 hydrophobin (125 amino acids) (Schuren and Wessels 1990|, and with the deduced amino acid sequence of the $A$. nidulans rodA gene product (157 amino acids) (Stringer et al. 1991). Alignments were generated by using the GCG gap program (Devereux et al. 1984) and were adjusted further by inspection. Amino acid identities are shown with vertical bars, and conserved substitutions are denoted by double dots. The eight conserved cysteine residues are boxed. $(B)$ Hydrophobicity plots of $\mathrm{Ccg}-2, \mathrm{Sc} 3$, and RodA proteins. Points above the $\mathrm{x}$-axis indicate hydrophobic regions; points below the $\mathrm{x}$-axis represent hydrophilic domains.

A

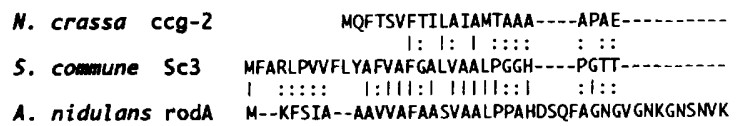

A. nidulans rodA M--KFSIA--AAVVAFAASVAALPPAHDSQFAGNGVGNGNSNWK

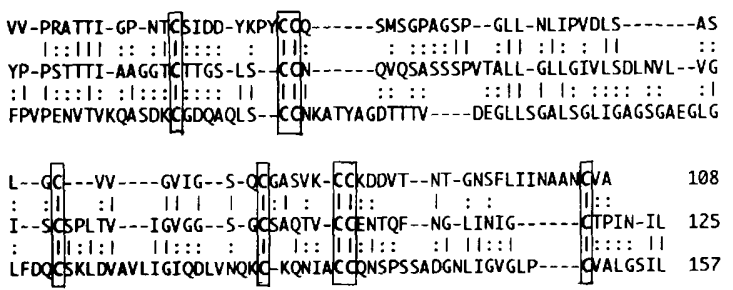

B

$\operatorname{ccg}-2$

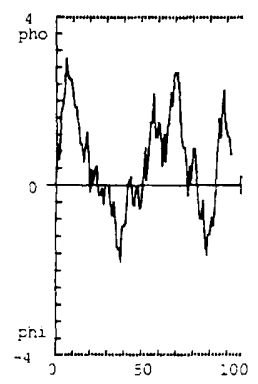

$\mathrm{Sc} 3$

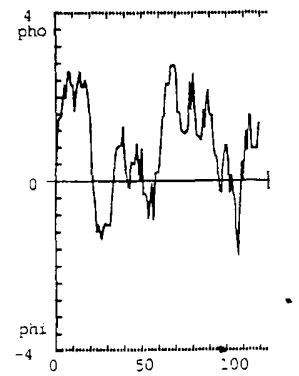

$\operatorname{rodA}$

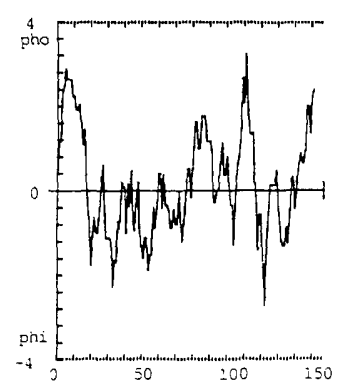

ค

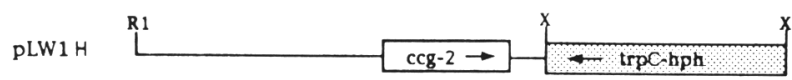

B

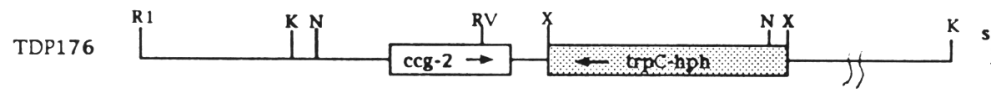

size
$\mathbf{k b}$

3.5

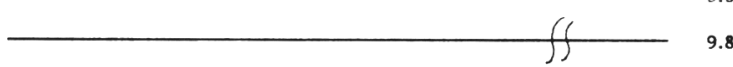

3.5
9.8

endogenous
ccg-2
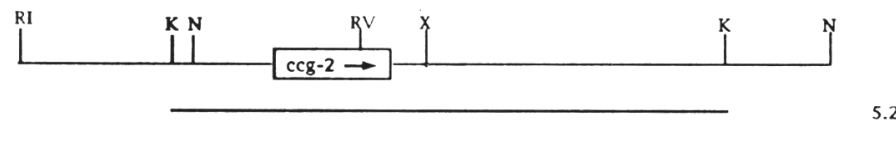

5.2

7.2

probe

Figure 4. Molecular analysis of eas complementation by $c c g-2$ DNA. (A) Constructs used for Southern analysis of eas-rescued transformant TDP176. pLW1H, used for ectopic transformation and rescue of easA, is shown with the relevant restriction enzyme sites. Both the $c c g-2$ transcriptional unit and the $t r p C-h p h$ cassette are boxed. Both TDP176-transformed DNA and the endogenous copy of $c c g-2$ are shown with the estimated sizes of the predicted fragments from the Southern analysis in $B$. Sequences extending $5^{\prime}$ or $3^{\prime}$ of pLW1H in TDP176 are unknown as a result of the ectopic nature of the chromosomal insertion. (R1) EcoRI; (K) KpnI; (N) NdeI; (RV) EcoRV; (X) XbaI. (B) Southern analysis of strains used in complementation assays of eas. Genomic DNA from strains bdA (control), TDP176, and easA (lanes 1-3, respectively) was digested with KpnI or NdeI. The DNA fragments were transferred to a nylon membrane and hybridized to a labeled ccg-2 NdeI-EcoRV probe (shown in $A$ ). The estimated sizes of the observed fragments are indicated and are correlated to the restriction map of TDP176 and to the endogenous $c c g-2$ region of the genome $(A)$. 
and $9.8 \mathrm{~kb}$ for $N d e \mathrm{I})$. The sizes of the additional bands are compatible with an ectopic integration event that did not interrupt the region of $\mathrm{pLW} 1 \mathrm{H}$ containing $c c g-2$ and the selectable hph marker. These data are consistent with expression of $c c g-2$ from the integrated pLW1H plasmid complementing the defect in the easA strain and restoring surface rodlets.

The presence of the rodlet layer in TDP176 was verified by transmission electron microscopy, performed on 13-day-old conidiating cultures, as described by Beever and Dempsey (1978). As shown, rodlet bundles are present in the control bdA strain (Fig. 5A), but absent (or in some replicas, greatly reduced to $<90 \%$ of $b d \mathrm{~A}$ ) in eas A (Fig. 5B). The rodlet layer was restored in TDP176 (Fig. 5C) but not in strains transformed with the vector alone (data not shown). Although these data suggest that ccg-2 and eas are the same gene and that Ccg- 2 encodes a necessary component of the rodlet layer, inactivation of $c c g$-2 was accomplished, as described below, to rule out the possibility that phenotype rescue resulted from ccg-2 encoding a closely linked suppressor of eas.

Inactivation of ccg- 2 by RIP results in an eas phenotype During the sexual phase of the $N$. crassa life cycle, duplicated genes are recognized and modified at frequencies ranging from about 10 to $100 \%$ by a process termed RIP (repeat induced point mutations) (Selker 1990). This mutational process provides a unique means of generat- ing a gene-specific null strain and was used to inactivate the $c c g-2$ locus as follows. The $1.9-\mathrm{kb} c c g-2 \mathrm{KpnI}-\mathrm{XbaI}$ fragment (Fig. 2) was inserted into vector pCSN43 (Staben et al. 1989) conferring hygromycin resistance, to generate plasmid $\mathrm{pLW} 1 \mathrm{KH}$. $N$. crassa bdA was transformed with pLW1KH, and the transformants were isolated on medium containing $200 \mu \mathrm{g} / \mathrm{ml}$ of hygromycin B. A putative homokaryotic transformant (TDP200) was obtained by conidial plating and was subsequently crossed to a strain of the opposite mating type, $b d a$.

The phenotype of the potential RIP ccg-2 progeny was examined, where $29 \%$ (63/214 progeny) appeared by several criteria to be identical to that of eas. First, conidia displayed the typical wetted dark orange appearance. Second, the conidia did not become airborne when agar slants were tapped. Both features are indicative of a lack of outer conidial rodlet fascicles. To confirm this, electron microscopy was performed on one of the ccg-2-inactivated progeny, TDP200-1. The results are shown in Figure 5D where, similar to most spores from eas A, the outer conidial rodlet layer is reduced to undetectable levels in the $c c g$-2 RIP strain.

ccg-2 transcript levels are greatly reduced in eas $A$ In addition to finding tight linkage between two mutations, similarities in mutant phenotypes, and complementation of the mutation in eas by a normal copy of $c c g-2$, an assertion of allelism between eas and ccg-2 would be
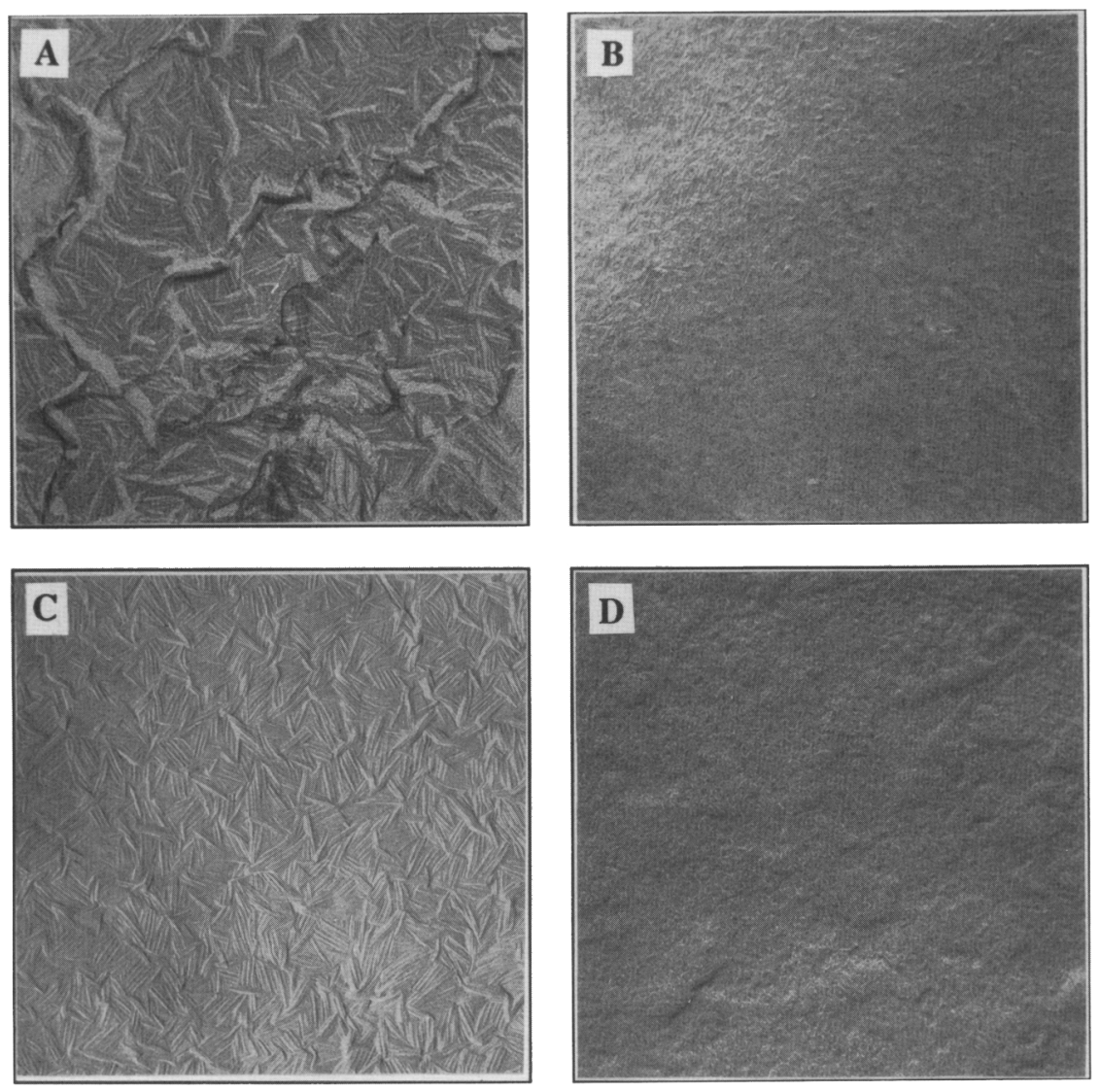

Figure 5. Transmission electron microscopy of conidial cell surface morphology in wild-type, mutant, transformed, and inactivated strains. Pictured are conidial replicas from 13-day-old slants of control strain bdA $(A)$, easA $(B), \operatorname{TDP} 176(C)$, TDP200-1 $(D)$, all examined by transmission electron microscopy and magnified at $25,000 \times$; each panel is $\sim 3.4 \mu \mathrm{m}$ on a side. Parallel bundles of rodlets are evident in both $b d A$ and TDP176 $(A, C)$, present but greatly diminished in eas $\mathrm{A}$, and altogether absent in the ccg-2-inactivated strain TDP200-1 $(B, D)$. 
supported by showing that the canonical eas mutation lies within the $c c g$-2 transcription unit or its cis-acting regulatory sequences. Partial DNA sequence analysis of the $c c g$-2 protein-coding region from eas (UCLA191), however, revealed no changes in the sequence /data not shown), suggesting that the defect lies in the elements regulating transcript abundance. To confirm this, total RNA was isolated from mycelia grown in liquid culture in constant darkness. Tissue from control bdA, easA, and TDP176 strains was harvested at times representing CT0, CT6, CT12, and CT18. RNA was transferred to nylon and probed with a ccg-2-specific RNA riboprobe (Fig. 6). Rhythmic expression of the 776 nucleotide $c c g-2$ message is observed in both bdA (Fig. 6A) and in the rescued TDP176 strain (Fig. 6C) (although observed to be slightly phase-shifted in TDP176) but reduced to barely detectable levels in eas A (Fig. 6B). Low levels of $c c g-2$ mRNA are evident at CT0 and CT18 only after extended exposure of the autoradiograph. As a control for circadian-regulated expression of RNA, the same membrane was probed with a different clock-regulated gene identified in N. crassa (D. Bell-Pedersen, unpubl.). This gene, $c c g-4$, encodes a message of $\sim 1.2 \mathrm{~kb}$, which accumulates at high levels at CT18-CTO. The ccg-4 transcript is present and correctly regulated in all three strains. The extremely low expression levels of ccg-2 mRNA in eas, together with the close genetic map positions, the complementation of eas with ccg-2 DNA, and the loss of rodlets in a ccg-2 RIP strain, provide additional evidence that $c c g-2$ and eas are allelic.

Identification of the eas defect In the course of examining eas DNA by Southern analysis, we observed that an EcoRV fragment generated from the ccg-2 locus was altered in size (data not shown). Double digestion of

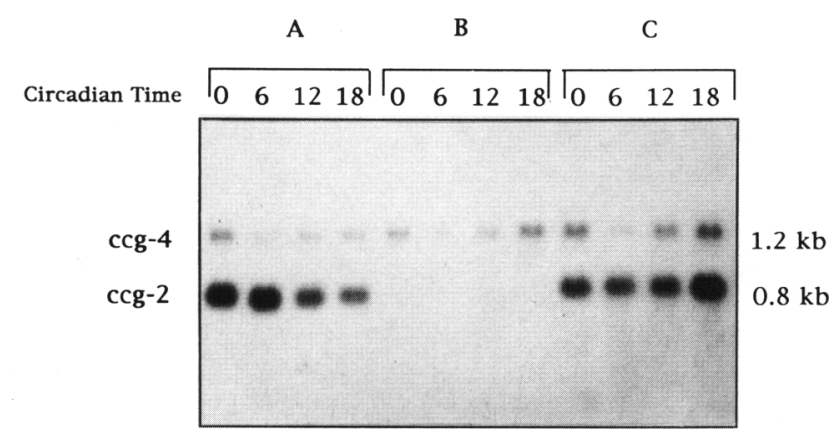

Figure 6. $c c g-2$ expression in mutant and rescued strains. Total RNA was isolated from mycelia harvested at CT0, CT6, CT12, and CT18 in control bdA $(A)$, easA $(B)$, and TDP176 $(C)$ strains. The RNA was transferred to nylon and probed with a $\left[{ }^{32} \mathrm{P}\right] \mathrm{UTP}$ labeled $c c g-2$ riboprobe. The membrane was subsequently hybridized with a riboprobe generated from $c c g-4$, a clock-regulated gene control (D. Bell-Pedersen, unpubl.). Both the $c c g-2$ $(0.8 \mathrm{~kb})$ and $c c g-4(1.2 \mathrm{~kb})$ transcripts are evident and under clock control in $A$ and $C$, whereas the $c c g-2$ transcript is absent in the eas background $(B)$. Limited amounts of $c c g-2$ message are, however, detectable at CT0 and CT18 after extended exposure.
easA, TDP176, and control bdA DNA with EcoRV and SalI was performed to determine which area of the $c c g-2$ locus was altered, as two EcoRV sites are present in this region. A 1.8 - $\mathrm{kb}$ upstream fragment present in both $b d \mathrm{~A}$ and $c c$-2-transformed TDP176 is absent in eas, whereas the downstream $0.5-\mathrm{kb}$ band is present in all three lanes, particularly after longer exposure times (Fig. 7A). This missing 1.8-kb fragment from eas coincides with the appearance of a larger $\sim 5-\mathrm{kb}$ band in both eas $\mathrm{A}$ and TDP176, consistent with an altered EcoRV site or the presence of an insertion upstream of the KpnI site. However, if the $E c o R V$ was specifically mutated, digestion of DNA with EcoRV and SalI should generate a fragment of $\sim 2.8 \mathrm{~kb}$ that utilizes an upstream SalI site; this 2.8-kb fragment is not observed in either eas $\mathrm{A}$ or TDP176. Also, in work reported previously elsewhere, when a strain carrying the eas mutation (UCLA191) is crossed, $\sim 20 \%$ of the progeny are observed to be deficient in cytochrome $a a_{1}$, the product of the subsequently identified unlinked cya-8 locus (Selker 1990; D. Perkins, pers. comm.). This observation led to the prediction that the eas mutation is caused by an insertion that includes cya- 8 sequences. Strains carrying the putative eas insertion mutation, when crossed, would be subject to inactivation of cya-8 by RIP. Thus, both the molecular and genetic data are consistent with the presence of an insertion.

To test for an upstream insertion, polymerase chain reaction (PCR) amplification was performed on genomic DNA isolated from easA, TDP176, and control strain bdA, using primers that span the $c c g-2$ locus (Fig. $7 \mathrm{~B}$ ). The results from three different amplifications are shown using oligodeoxynucleotide pairs: (I) oligos 01 and 010; (II) oligos 05 and 011; and (III) oligos 013 and 016 (for I, II, III, see Fig. 7B). As predicted, by using oligodeoxynucleotides that encompass the ccg-2 transcriptional unit (I and II) the sizes of the PCR products appear to be identical in all three strains. However, PCR products generated from the upstream region (III) of eas DNA are absent, whereas a 1.7-kb band is synthesized from control bdA and in ccg-2-transformed TDP176 DNA templates. Although we expected to observe a larger band from eas DNA and TDP176 as the result of an insertion, we were unable to detect this fragment directly or by probing the PCR products with upstream $c c g-2$ sequences under any conditions tested (possibly owing to the large size of the amplicon or disruption of the oligodeoxynucleotide-binding site). The lack of the $1.7-\mathrm{kb}$ band in eas is, however, consistent with the Southern analysis described above and, together, point to the presence of an insertion located upstream of the $c c g-2$ transcription unit.

The circadian clock is unaffected in strains lacking ccg2 The circadian clock is thought to be comprised of a feedback loop that allows for coordinate regulation of metabolic behavior and time perception (Dunlap 1990). Because in more complex systems it is known that clock-driven output can feed back to modulate the clock itself (e.g., Mrosovsky and Salmon 1987), it was of inter- 
A

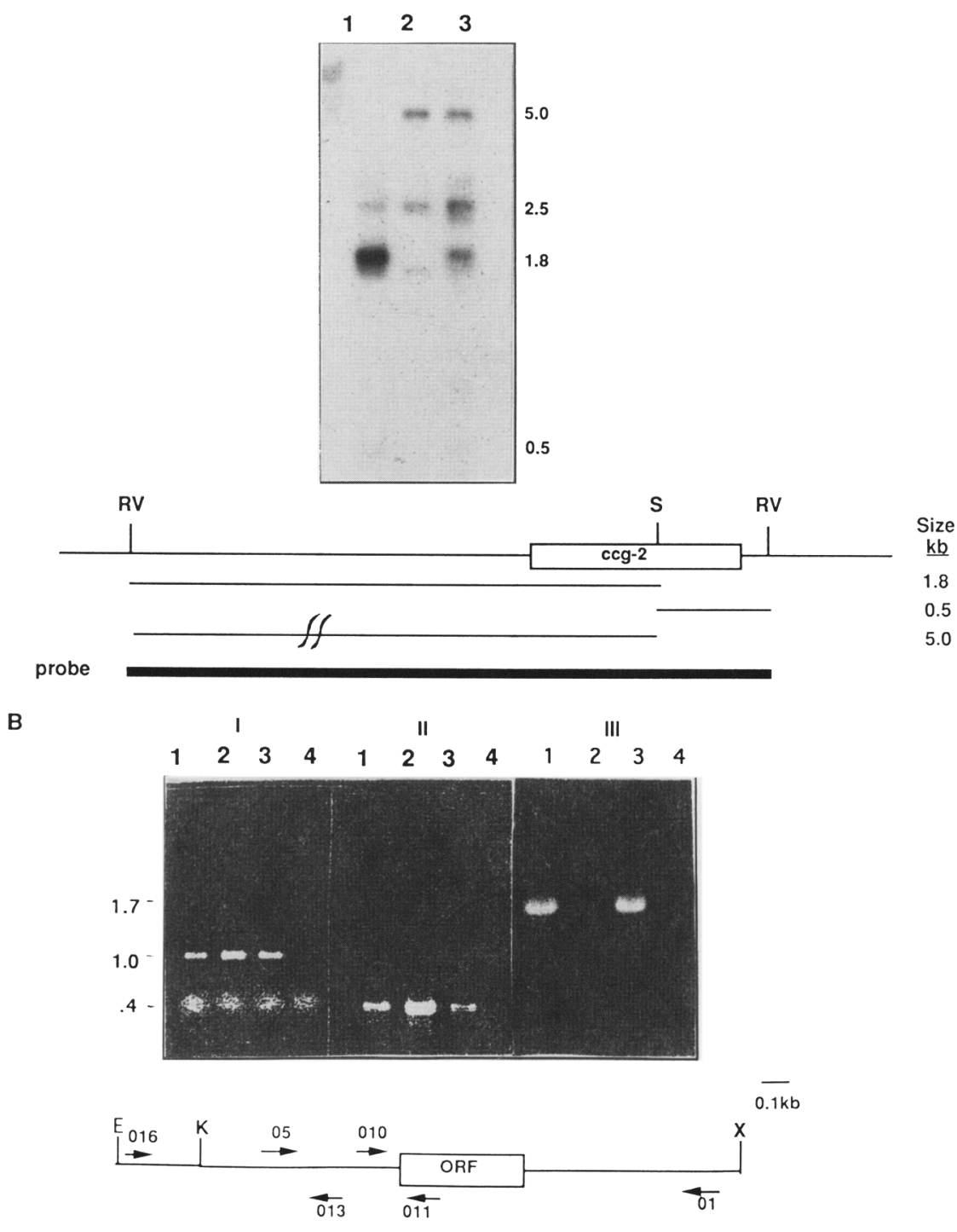

Figure 7. Molecular analysis of the eas defect. (A) Southern analysis of genomic DNA isolated from control strain $b d A$ (lane 1), easA (lane 2), and TDP176 (lane 3). DNAs were double-digested with EcoRV (RV) and SalI (S), transferred to nylon, and probed with a $2.2-\mathrm{kb}$ radiolabeled ccg-2 EcoRV fragment (shown below). The approximate sizes of the expected fragments are 1.8 and $0.5 \mathrm{~kb}$. The lack of detection of the $1.8-\mathrm{kb}$ band in eas A (lane 2) indicates the presence of a sequence aberration upstream of the $c c g-2$ transcription unit generating a larger fragment of $\sim 5.0$ $\mathrm{kb}$. This $5.0-\mathrm{kb}$ band is also observed in TDP176. The 0.5-kb fragment is evident in each lane after long exposure times (data not shown). The origin of the $2.5-\mathrm{kb}$ band observed in each lane is attributable to incomplete digestion with Sall. (B) PCR products were generated in the $c c g-2$ region using a combination of oligodeoxynucleotides $01 / 010$ (I), 05/011 (II), or 013/016 (III) (Materials and methods). Together, these pairs of oligodeoxynucleotides encompass all of the $c c g$-2-coding region and the $5^{\prime}$ upstream sequences as indicated on the map below. (K) KpnI; (X) XbaI; ORF (ccg-2 ORF). The DNA templates for PCR were $b d A$, easA, TDP176, and a no DNA control (lanes 1-4, respectively). Fragment sizes are indicated (in $\mathrm{kb})$ at left. est to determine whether lesions of $c c g$-2 could affect the clock circuit. Hence, we investigated clock function in a ccg-2 mutant background by analyzing clock-regulated conidial banding (Feldman and Hoyle 1976; Dunlap 1990). eas A was first crossed to bdA, which enhances assessment of the banding pattern on race tubes. Figure 8 shows the results of the clock assay for $b d A$, and $b d_{i}$ eas A strains. We found that $b d_{\text {; }} e a s$ conidia display an eas phenotype, that the period lengths for $b d \mathrm{~A}$ (top) and

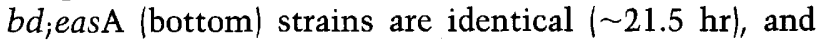
that the phase of the rhythm is unaffected by the eas mutation.

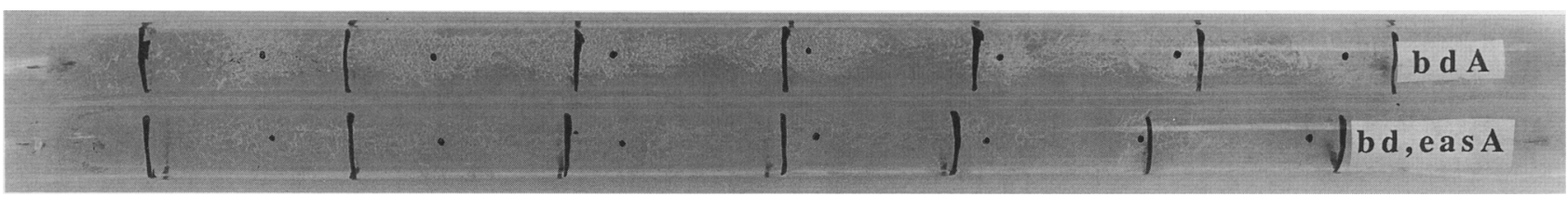

Figure 8. Clock control of conidiation. Race tube assay of clock control in bdA (top), and bd;easA (bottom). The leftward-most vertical black bar on each tube corresponds to the position of the growth front at the time of the light-to-dark transfer. Subsequent vertical black bars correspond to the hyphal growth front marked at $24-\mathrm{hr}$ intervals. Because linear growth rate is essentially constant throughout the day, horizontal distance is equivalent to hours in constant darkness following the light-to-dark transition. (O) The center of the conidial band on each day. 


\section{Discussion}

The mechanism by which the circadian clock controls cellular metabolism, one important feature of which is clock control of mRNA abundance, constitutes one of the least studied aspects of circadian biology. In attempts to begin characterization of clock output pathways, clock-regulated genes were targeted for isolation by subtractive hybridization (Loros et al. 1989), and two genes, $c c g-1$ and $c c g-2$, were identified. Both are abundantly expressed and transcriptionally activated specifically in the early subjective morning through a mechanism ultimately directed by the circadian clock (Loros and Dunlap 1991). Our initial focus in the analysis of these genes revolved around determining their sequence, structure, and function. To this end, $c c g-2$ has been shown here to encode a mature message of 776 nucleotides, which specifies an ORF of 108 amino acids (Figs. 1B and 2).

The close genetic linkage of a previously identified Neurospora developmental locus, eas, with ccg-2 (linkage group II R; Perkins 1990), the similarity of phenotype between alleles of eas and the $A$. nidulans developmental gene $\operatorname{rod} A$, and the homology of $\mathrm{Ccg}-2$ to the $A s$ pergillus rodlet protein RodA suggested that $c c g-2$ and eas were allelic. This was verified experimentally by showing that $c c g-2$ DNA complements the eas mutant strain and restores the rodlet layer on conidial surfaces (Fig. 5C). Additionally, we have demonstrated here that inactivation of the $c c g-2$ gene by RIP results in strains with an eas phenotype, including the lack of the conidial rodlet fascicles (Fig. 5D). Although it was reported originally that the eas mutant strain, allele UCLA191, completely lacked rodlet bundles (Beever and Dempsey 19781, we observed that some conidia had bundles present at low levels $<10 \%$ of wild-type levels, e.g., see Fig. 5B). This is consistent with the small amount of ccg-2 mRNA produced from the eas mutant allele, indicating that the eas defect is leaky. Preliminary investigations into the molecular defect in the original eas mutant strain points to a large insertion occurring upstream of the $c c g-2$ transcription unit. It was speculated previously (Selker 1990; D. Perkins, pers. comm.) that this insertion contained sequences of the cya-8 gene, as evidenced by inactivation of the cya- 8 gene in progeny from crosses with eas (UCLA191). Although the exact location or nature of the insertion was not determined in this study, we believe it occurs at least 625 nucleotides upstream of the ccg-2 transcriptional unit based on Southern and PCR analysis of eas DNA (Fig. 7). These results indicate the presence of a cis-acting sequence element required for $c c g-2$ expression located far upstream of the ccg-2-coding region. Two additional pieces of evidence support this hypothesis. First, we were unable to detect rescue of eas with just the 1.9-kb ccg-2 KpnI-XbaI fragment, even though this DNA fragment encodes the entire $c c g-2$ transcriptional unit and contains 625 nucleotides of $5^{\prime}$ sequences. Second, in initial experiments aimed at dissecting the ccg-2 cis-acting control elements, we observed that sequences upstream of the KpnI site are required for expression of $c c g-2$ mRNA (D. Bell-
Pedersen, unpubl.). Together, this information points to sequences upstream of $c c g-2$ being essential for normal levels of transcription. It appears, though, that $c c g-2$ expression is simply a driven output of the clock, because defects in expression have no apparent effect on the operation of the pacemaker itself (Fig. 8).

The clock-regulated $c c g-2$ gene encodes a characteristic fungal hydrophobin, a class of low-molecular-weight, hydrophobic proteins essential for establishment of the rodlet structures that are a critical element contributing to spore wall hydrophobicity and, therefore, ultimately spore dispersal. Typically, members of this class of developmentally regulated fungal proteins share a number of common characteristics including eight conserved cysteines, a putative signal sequence for secretion, and a strong hydrophobic amino terminus and central domain, characteristics shared by the polypeptides encoded by ccg-2, $\operatorname{rodA}, S c 1, S c 3$, and Sc4 (Fig. 3A; Schuren and Wessels 1990; Stringer et al. 1991). Rodlets are present on the surface of aerial cells in many filamentous fungal /Cole et al. 1979/ and bacterial species that elaborate dry spores (Holt and Leadbetter 1969; Williams et al. 1972). In Neurospora, rodlets are arranged in bundles, or fascicles, where individual chains are laid down in a parallel fashion within a single fascicle. Interdigitation of adjacent fascicles on the cell surface results in an interwoven appearance (Fig. 5). The conserved cysteine residues may be involved in the formation of disulfide bonds necessary for cross-linking the individual components.

We have noted previously that the coincidence of light and clock regulation in the $c c g$ genes may be illustrative of an emerging theme in this area of research (Dunlap 1990; G. Arpaia, J. Loros, J.C. Dunlap, and G. Macino, in prep.). In plants, a number of genes are known to be light regulated and circadianly regulated (e.g., Giuliano et al. 1988; for review, see Kay and Millar 1992). In vertebrate systems, several retinal photoreceptor components oscillate (e.g., Korenbrot and Fernald 1989), and in the vertebrate suprachiasmatic nucleus, light induces the expression of a number of immediate early genes in a time-ofday-specific manner (Aronin et al. 1990; Earnest et al. 1990; Kornhauser et al. 1990; Rusak et al. 1990; for review, see Taylor 1990). In fungi, a number of processes are known to be dependent on or influenced by blue light (for review, see Degli-Innocenti and Russo 1984a), and this represents a focus of work in Neurospora. For instance, in the context of sexual reproduction, light enhances the production of protoperithecia (Degli-Innocenti and Russo 1984b), which themselves display a positive phototropism (Harding and Melles 1983), and ascospore release from the mature perithecium follows a clearly defined circadian rhythm in both $N$. crassa (S. Brody, unpubl. in Lakin-Thomas et al. 1990) and Neurospora tetrasperma (R. Brambl, pers. comm.). This interplay between light regulation and clock regulation may provide some insight into the adaptation of the fungus to its ecological niche.

Neurospora species are typically found in warm temperate or semitropical environments, where the breezes necessary for efficient spore dispersal occur most de- 
pendably at the times of maximum temperature differential between air and land-in the morning and evening. Conidia elaborated in the middle of the day for dispersal in the evening would be subject to desiccation and UV mutation for hours before the evening. However, if conidial development were to be initiated in the late night in anticipation of dawn, initially triggered by an endogenous clock and further enhanced by light at dawn, then conidia might be present to be carried off by morning winds before the heat of midday. Clock control of this event may therefore represent a practical evolutionary adaptation to ensure that spores are prepared for dissemination at the proper time of day in the wild. It is reasonable to assume that genes involved in the conidiation process, including conidial maturation (such as ccg-2) are under the control of the circadian clock to allow for coordinate expression at the proper time of day. In this regard, it is of interest to note that the upstream region of $c c g-2$ was found to share extensive sequence homology to a conidiation gene, con 10 , from N. crassa (Roberts et al. 1988) as well as to regions of the ccg-1 gene (data not shown). The functions of both con10 and ccg-1 are not yet known.

Light is important for conidiation in many strains of fungi, including the chestnut blight fungus Cryphonectria parasitica, A. nidulans (Mooney and Yager 1990), and for the formation of fruiting bodies in Schizophyllum (Perkins 1969); yet, all of these fungi grow well in the absence of light. It thus seems likely that light is being used as an overt environmental cue to stimulate development at appropriate times; hence, it seems likely that circadian rhythms may be found in these and other systems to be a covert cue, initiating aspects of growth and development in the absence of light and potentiating the developmental system to respond to light. Recalling that the overt circadian banding rhythm of Neurospora is obscured in wild-type strains by the levels of $\mathrm{CO}_{2}$ built up in petri dishes or race tubes under normal culture conditions (hence, the use of the band mutation to relieve this $\mathrm{CO}_{2}$ masking effect), it is possible that rhythms in other fungi may heretofore have been overlooked owing to similar masking problems. However, the present study suggests an alternate screen for rhythmicity. Because similar spore surface hydrophobins have been found in several fungi to date and appear to repre- sent a class of proteins common to most fungi that produce aerial hyphae and spores and because a hydrophobin gene from Neurospora, ccg-2, is clearly under circadian regulation, analysis of the temporal regulation of hydrophobin genes from other fungal species might be a good place to initiate a search for a molecular correlate of the circadian clock in any novel species in which a clock has not been described previously. One would thus predict that the Sc genes of Schizophyllum and the $\operatorname{rodA}$ gene of Aspergillus might display a rhythm in their level of expression.

The regulation of the $c c g-2$ gene appears quite complex. Not only is the gene under clock control, but expression is induced by light. Transcripts from ccg-2 are present in germinating conidia (where levels are not constitutive, but fluctuate; K.M. Lindgren, unpubl.), and the gene is regulated along with the conidiation process. The molecular analysis of $c c g-2$ described here will facilitate future studies aimed at determining how these disparate factors responsible for regulation of $c c g$-2 interdigitate at the single gene level. Additionally, this gene and the other ccgs will provide a useful entre into the cis- and trans-acting elements responsible for clock-regulated expression in attempts to identify and define the individual components of the clock output pathway.

\section{Materials and methods}

Neurospora strains and growth conditions

Strains of $N$. crassa used in this study are listed in Table 1. Growth media (Vogel's and Fries minimal media), vegetative growth conditions, and crossing protocols are fully described in Davis and deSerres (1970). Transformation of $N$. crassa spheroplasts with plasmids containing the $h p h$ gene as a selectable marker was accomplished according to the standard protocol of Vollmer and Yanofsky (1986). Transformants were selected on medium containing $200 \mu \mathrm{g} / \mathrm{ml}$ of hygromycin B (CalBiochem).

\section{Plasmids and oligodeoxynucleotides}

The $c c g$-2 gene used to generate plasmids described in this study originated from a $\lambda J 1$ genomic clone of $\sim 13 \mathrm{~kb}(7 \mathrm{Cl}$; Loros et al. 1989|. Plasmid pS7C1 harbors a 6-kb ccg-2 XbaI fragment isolated from $\lambda 7 \mathrm{Cl}$ (shown in Fig. $1 \mathrm{~B}$ ) and inserted into the $X b a \mathrm{I}$ site of pBluescript $+(\mathrm{pBSK}+$, Stratagene $)$. Deletion of an upstream 3-kb EcoRI fragment from $\mathrm{pS} 7 \mathrm{Cl}$ yielded plasmid $\mathrm{pLW} 1$

Table 1. Neurospora strains used in this study

\begin{tabular}{llll}
\hline Strain name & Strain no. & Genotype & Source \\
\hline 74-OR23-VA & & St. Lawrence wild-type isolate & FGSC $^{\text {a } 2489}$ \\
bdA & $30-7$ & bandA & Sargent et al. (1966) \\
bda & $87-3$ & banda & Sargent et al. (1966) \\
easA & UCLA191 & easA (UCLA191) & FGSC 2960 \\
bd;easA & $101-4$ & band,easA & this work \\
TDP176 A & & eas pLW1H transformant & this work \\
TDP200 A & & bandA pLW1KH transformant & this work \\
TDP200-1 & & TDP200 A $\times$ banda ccg-2 RIP progeny & this work \\
\hline
\end{tabular}

${ }^{\text {a} F u n g a l ~ G e n e t i c s ~ S t o c k ~ C e n t e r, ~ U n i v e r s i t y ~ o f ~ K a n s a s ~ M e d i c a l ~ C e n t e r, ~ K a n s a s ~ C i t y, ~ K a n s a s . ~}$

bUCLA191 was the only isolate of eas before the RIP progeny described in this study. 
(containing $3 \mathrm{~kb}$ of the $c c g-2$ locus). Insertion of the $\sim 2-\mathrm{kb}$ trp C$h p h$ XbaI cassette from pCSN43 (Staben et al. 1989) into the unique $X b a I$ site of pLWl generated plasmid pLW1H (Fig. 4). pLW1K, which contains all of the ccg- 2 transcription unit, was constructed by deletion of a 1.1-kb KpnI fragment from pLW1. Insertion of the $\operatorname{trpC}-h p h \mathrm{XbaI}$ cassette from pCSN43 into pLWlK yielded plasmid $\mathrm{pLW} 1 \mathrm{KH}$, which was used for transformation of $b d A$ to inactivate $c c g-2$ through RIP. All plasmids were generated using standard techniques (Sambrook et al. 1989). Restriction enzymes were obtained from New England Biolabs and used according to the manufacturer's instructions. Plasmids and M13 phages were propagated in Escherichia coli strains XL1 Blue or JM101.

Oligodeoxynucleotides (with map locations on the ccg-2 sequence indicated in parentheses/ used in this study were synthesized on an Applied Biosystems synthesizer and include 01, 5'-TAC CGC CTT TTG CCT-3' (1103-1089); 05, 5'-TGT CAC AAT TCT TGA TCG-3' (-262--243); 010, 5'-CTC CCA GCT TCT TTC AGC-3' (78-95); 011, 5' -TGA AGA CGC TGG TGA ACT-3' (135-118); 013, 5'-ACA CTC GTC ATT TCC AGA T-3' $(-170-189)$; and 016, 5'-ACA TGT GGT ACG TAT GAC T-3' (located $\sim 1.5-\mathrm{kb}$ upstream of the $5^{\prime}$ sequenced region shown in Fig. 2).

\section{Nucleic acid sequencing and protein sequence comparisons}

pLW1K was used as a template for double-stranded DNA sequencing of miniprep DNA by dideoxynucleotide chain-termination techniques (Sanger et al. 1977) using a modified T7 bacteriophage DNA polymerase (Sequenase, U.S. Biochemical). The sequence of both strands was determined. Sequencing of single-stranded DNA templates isolated from $\mathrm{Ml} 3 \mathrm{ccg}-2$ clones was by the same method and was used to confirm the doublestranded DNA sequence results. ccg-2 cDNA isolated from a $\lambda$ gt10 library (Fu and Marzluff 1990) was used as a PCR template for sequencing. PCR products generated from $\lambda g t 10-c c g-2$ cDNA were precipitated in 0.6 volume of $20 \%$ PEG and $2.5 \mathrm{M}$ $\mathrm{NaCl}$ and were sequenced as above.

The predicted Ccg-2 protein sequence was compared with translated sequences from NBRF-PIR protein (release 29.0) and SWISS-PROT (release 19.0), using the University of Wisconsin Genetics Computer Group (GCG) FASTA sequence analysis programs (Devereux et al. 1984). Hydrophobicity plots were generated with the MacPROT/hyd program based on the KyteDoolittle algorithm, with a moving window of 7 residues (Kyte and Doolittle 1982). Potential sites of phosphorylation and myristylation were identified using MacPattern 1.5 (Fuchs 1991) utilizing the PRO.SITE data base release 8 .

\section{Nucleic acid isolation, radioactive probes, and hybridization}

Genomic DNA was isolated from $N$. crassa mycelia grown in $1 \times$ Vogel's salts containing $2 \%$ glucose by the CTAB method (Zolan and Pukkila 1986; Taylor and Natvig 1987). Approximately $1 \mu \mathrm{g}$ of DNA was digested, fractionated on a $1 \%$ agarose gel, and transferred to nylon membrane (Hybond-N, Amersham), and the blots were processed by standard techniques (Sambrook et al. 1989). DNA probes for Southern analyses were labeled by the random priming method of Feinberg and Vogelstein (1983). The probes included a 1.2-kb KpnI-EcoRV ccg-2 fragment (Fig. 4) or a 2.3-kb EcoRV ccg-2 fragment (Fig. 7), both of which were derived from plasmid pLWl.

RNA was isolated by the method of Reinert et al. (1981) from clock-synchronized mycelia grown in liquid culture without shaking (Loros et al. 1989; Loros and Dunlap 1991). Light-todark transfer times were such that the ages of the cultures at harvest were approximately the same, but the circadian times varied according to the following schedule. Tissue for RNA extraction was harvested after 8 (CT21), 12 (CT1), 18 (CT8), 22 (CT12), 25 (CT16), 32 (CT0), and 36 (CT4) hr in the dark (Fig.1), or 16 (СT6), 22 (СT12), 27 (CT18), and 32 (СT0) hr in the dark (Fig. 6). Ten micrograms of total RNA was separated on a $1 \%$ agarose-formaldehyde gel (Lehrach et al. 1977), transferred to nitrocellulose membranes (Nitropure, MSI), and probed with a ${ }^{32}$ P-labeled $c c g-2 \mathrm{KpnI}-\mathrm{XbaI}$ DNA probe (Fig. 1) or with antisense RNA probes (riboprobes) (Fig. 6). Riboprobes were synthesized from a T3 promoter located in the $\mathrm{pBSK}^{+}$vector, using T3 RNA polymerase (Promega) in the presence of $\left[{ }^{32} \mathrm{P}\right] \mathrm{UTP}$ as described by Promega. Hybridization and washing conditions were performed as in Sambrook et al. (1989) for DNA probes or as recommended in the Promega Protocols and Applications guide (1991) for RNA probes.

\section{PCR amplification}

$\lambda$ gt 10 ccg-2 cDNA clones were amplified for dideoxynucleotide sequencing using Taq polymerase (Boehringer Mannheim) from a one-tenth dilution of a high titer $\left(10^{12} \mathrm{pfu} / \mathrm{ml}\right)$ lysate that was heated for $10 \mathrm{~min}$ at $70^{\circ} \mathrm{C}$. One microliter of the phage dilution was used for standard PCR reactions. PCR amplification of $\mathrm{Neu}$ rospora genomic DNA from bdA, easA, and TDP176 ( 100 ng) was accomplished using 10 pmoles of each primer in buffer containing $300 \mu \mathrm{M}$ of each dNTP. Amplification was achieved by using 35 cycles of a $30-\mathrm{sec}$ denaturation at $94^{\circ} \mathrm{C}$, a $30-\mathrm{sec}$ annealing at $45^{\circ} \mathrm{C}$, a 3 -min extension at $72^{\circ} \mathrm{C}$, followed by a final 7 -min extension at $72^{\circ} \mathrm{C}$. PCR products were separated on a $1 \%$ agarose gel containing $10 \mu \mathrm{g} / \mathrm{ml}$ of ethidium bromide.

\section{Electron microscopy}

Replicas of the surface of conidia from strains bdA, easA, TDP176, and TDP200-1 (Fig. 5) were prepared from 13-day-old slants as described by Beever and Dempsey (1978). Conidia were tapped onto thin glass disks, vacuum evaporated in a Balzers 301 evaporator, and shadowed at $45^{\circ} \mathrm{C}$ with carbon and platinum. A backing layer of carbon was then added at $90^{\circ} \mathrm{C}$. The replicas were floated onto water, treated with chromic acid for $2 \mathrm{hr}$, followed by overnight washing in bleach to remove any remaining conidial material. Replicas were then washed in several changes of water, picked up on grids, and examined by transmission electron microscopy using a JEOL $100 \mathrm{CX}$ microscope.

\section{Acknowledgments}

We gratefully acknowledge Charles Daghlian and Louisa Howard for assistance with the electron microscopy, Keith Johnson for aid in computer sequence analysis, and Lori White for construction of pLW1. In addition, we thank members of our laboratory, Ben Aronson, Norman Garceau, Keith Johnson, Kristin Lindgren, and Martha Merrow, for technical assistance and advice. This work was supported by grants from the National Science Foundation (DMB 8819957), the National Institute of Mental Health (MH 44651), and the Norris Cotton Cancer Center at Dartmouth Medical School.

The publication costs of this article were defrayed in part by payment of page charges. This article must therefore be hereby marked "advertisement" in accordance with 18 USC section 1734 solely to indicate this fact.

\section{Note}

During the preparation of this manuscript describing the allelism of $c c g-2$ and eas, we became aware that a manuscript de- 
scribing allelism of eas with another gene was ready for submission (see Lauter et al., this issue).

\section{References}

Aronin, N., S. Sager, F. Sharp, and W. Schwartz. 1990. Light regulates expression of a Fos-related protein in rat suprachiasmatic nuclei. Proc. Natl. Acad. Sci. 87: 5959-5963.

Beever, R.E. and G.P. Dempsey. 1978. Function of rodlets on the surface of fungal spores. Nature 272: 608-610.

Beever, R.E., R.J. Redgwell, and G.P. Dempsey. 1979. Purification and chemical characterization of the rodlet layer of Neurospora crassa conidia. J. Bacteriol. 140: 1063-1070.

Boyd, D. and J. Beckwith. 1990. The role of charged amino acids in the localization of secreted and membrane proteins. Cell 62: 1031-1033.

Cole, G.T., S. Takashi, R. Kasai, T. Yokoyama, and Y. Nozawa. 1979. Surface ultrastructure and chemical composition of the cell walls of conidial fungi. Exp. Mycol. 3: 132-156.

Cullen, D., S. Leong, L.J. Wilson, and D.J. Henner. 1987. Transformation of Aspergillus nidulans with the hygromycin resistance gene, $h p h$. Gene 57: 21-26.

Davis, R.H. and F.J. deSerres. 1970. Genetic and microbiological research techniques for Neurospora crassa. Methods Enzy. mol. 17A: 79-143.

Degli-Innocenti, F. and V.E.A. Russo. 1984a. Genetic analysis of blue-light induced responses in Neurospora crassa. In Blue light effects in biological systems (ed. H. Senger), pp. 214 219. Springer-Verlag, Berlin.

- 1984b. Isolation of new white collar mutants of Neurospora crassa and studies on their behavior in blue lightinduced formation of protoperithecia. J. Bacteriol. 139: 757761.

Dempsey, G.P. and R.E. Beever. 1979. Electron microscopy of the rodlet layer of Neurospora crassa conidia. I. Bacteriol. 140: 1050-1062.

Devereux, J., P. Haeberli, and O. Smithies. 1984. A comprehensive set of sequence analysis programs for the VAX. Nucleic Acids Res. 12: 387-395.

Dunlap, J.C. 1990. Closely watched clocks: Molecular analysis of circadian rhythms in Neurospora and Drosophila. Trends Genet. 6: 159-165.

Earnest, D.J., M. Iadarola, H.H. Yeh, and J.A. Olschowka. 1990. Photic regulation of c-fos expression in neural components governing entrainment of circadian rhythms. Exp. Neurol. 109: 353-361.

Edmunds, L.N. 1988. Cellular and molecular bases of biological clocks. Springer-Verlag, New York.

Feinberg, A.P. and B. Vogelstein. 1983. A technique for radiolabeling DNA restriction endonuclease fragments to high specific activity. Anal. Biochem. 132: 6-13.

Feldman, J.F. and M.N. Hoyle. 1976. Complementation analysis of linked circadian clock mutants of Neurospora crassa. Genetics 82: 9-17.

Feldman, J.F. and I.C. Dunlap. 1983. Neurospora crassa: A unique system for studying circadian rhythms. Photochem. Photobiol. Rev. 7: 319-368.

Fu, Y.-H. and G. A. Marzluff. 1990. nit-2, the major nitrogen regulatory gene of Neurospora crassa, encodes a protein with a putative zinc finger DNA-binding domain. Mol. Cell. Biol. 10: $1056-1065$.

Fuchs, R. 1991. MacPattern 1.5. EMBL Data Library, Heidelburg, Germany.

Guiliano, G.N., N.E. Hoffman, K. Ko, P.A. Scolnic, and A.R. Cashmore. 1988. A light entrained circadian clock controls transcription of several plant genes. EMBO f. 7: 3635-3642.

Gurr, S.J., S.E. Unkles, and J.R. Kinghorn. 1987. The structure and organization of nuclear genes of filamentous fungi. In Gene structure in eukaryotic microbes (ed. J.R. Kinghorn), pp. 93-139. IRL Press, Oxford, England.

Harding, R.W. and S. Melles. 1983. Genetic analysis of phototropism of Neurospora crassa perithecial beaks using white collar and albino mutants. Plant Physiol. 72: 996-1000.

Hawker, L.E. and M.F. Madelin. 1976. The dormant spore. In The fungal spore: Form and function (eds. D.J. Weber and W.M. Hess), pp. 1-70. Wiley, New York.

Holt, S.C. and E.R. Leadbetter. 1969. Comparative ultrastructure of selected aerobic spore forming bacteria: A freezeetching study. Bacteriol. Rev. 33: 346-378.

Kay, S. and A. Millar. 1992. Circadian regulated Cab gene expression in higher plants. In The molecular biology of circadian rhythms (ed. M. Young). Marcel Dekker, New York. (In press.)

Korenbrot, J.L. and R.D. Fernald. 1989. Circadian rhythm and light regulate opsin mRNA in rod photoreceptors. Nature 337: 454-457.

Kornhauser, J., D. Nelson, K. Mayo, and J. Takahashi. 1990. Photic and circadian regulation of $c$-fos gene expression in the hamster suprachiasmatic nucleus. Neuron 5: 127-134.

Kyte, J. and R.F. Doolittle. 1982. A simple method for displaying the hydropathy character of a protein. I. Mol. Biol. 157: 105132.

Lakin-Thomas, P.L., G.G. Cote, and S. Brody. 1990. Circadian rhythms in Neurospora crassa: Biochemistry and genetics. Crit. Rev. Microbiol. 17: 365-416.

Lehrach, H., D. Diamond, J.M. Wozney, and H. Boedtker. 1977. RNA molecular weight determinations by gel electrophoresis under denaturing conditions, a critical reexamination. Biochemistry 16: 4743-4751.

Loros, J.J. and J.C. Dunlap. 1991. Neurospora clock-controlled genes are regulated at the level of transcription. Mol. Cell. Biol. 11: 558-563.

Loros, J.J., S.A. Denome, and J.C. Dunlap. 1989. Molecular cloning of genes under control of the circadian clock in Neurospora. Science 243: 385-388.

McClung, C.R., B.A. Fox, and J.C. Dunlap. 1989. The Neurospora clock gene frequency shares a sequence element with the Drosophila clock gene period. Nature 339: 558-562.

Mooney, J.L. and L.N. Yager. 1990. Light is required for conidiation in Aspergillus nidulans. Genes \& Dev. 4: 1473-1482.

Mrosovsky, N. and P. Salmon. 1987. A behavioral method for accelerating re-entrainment of rhythms to new light-dark cycles. Nature 330: 372-373.

Perkins, J.H. 1969. Morphogenesis in Schizophyllum commune. I. Effects of white light. Plant Physiol. 44: 1706-1712.

Perkins, D.D. 1990. Neurospora crassa genetic maps, June 1989. Fungal Genet. Newslett. (Suppl.) 37: 138-147.

Reinert, W.R., V.B. Patel, and N.H. Giles. 1981. Genetic regulation of the qa gene cluster of Neurospora crassa: Induction of qa messenger ribonucleic acid and dependency on qa-1 function. Mol. Cell. Biol. 1: 829-835.

Roberts, A.N., V. Berlin, K.M. Hager, and C. Yanofsky. 1988. Molecular analysis of a Neurospora crassa gene expressed during conidiation. Mol. Cell. Biol. 6: 2411-2418.

Rusak, B., H. Robertson, W. Wisden, and S. Hunt. 1990. Light pulses that shift rhythms induce gene expression in the suprachiasmatic nucleus. Science 248: 1237-1239.

Sambrook, I., E.F. Fritsch, and T. Maniatis. 1989. Molecular cloning: A laboratory manual, 2nd ed. Cold Spring Harbor Laboratory Press, Cold Spring Harbor, New York.

Sanger, F., S. Nicklen, and A.R. Coulson. 1977. DNA sequenc- 
Bell-Pedersen et al.

ing with chain-terminating inhibitors. Proc. Natl. Acad. Sci. 74: 5463-5467.

Sargent, M.L., W.R. Briggs, and D.O. Woodward. 1966. The circadian nature of a rhythm expressed by an invertaseless strain of Neurospora crassa. Plant Physiol. 41: 1343-1349.

Schuren, F.H.J. and J.G.H. Wessels. 1990. Two genes specifically expressed in fruiting dikaryons of Schizophyllum commune: Homologies with a gene not regulated by mating-type genes. Gene 90: 199-205.

Selitrennikoff, C.P. 1976. Easily-wettable, a new mutant. Neurospora Newslett. 23: 23.

Selker, E.U. 1990. Premeiotic instability of repeated sequences in Neurospora crassa. Annu. Rev. Genet. 24: 579-613.

Staben, C., B. Jensen, M. Singer, J. Pollock, M. Schechtman, J. Kinsey, and E.U. Selker. 1989. Use of bacterial hygromycin B resistance gene as a dominant selectable marker in Neurospora crassa transformation. Fungal Genet. Newslett. 36: $79-81$.

Stringer, M.A., R.A. Dean, T.C. Sewall, and W.E. Timberlake. 1991. Rodletless, a new Aspergillus developmental mutant induced by directed gene inactivation. Genes \& Dev. 5: 1161-1171.

Taylor, R. 1990. Immediate-early genes may offer a new key to the circadian clock. $J$. NIH Res. 2: 49-53.

Taylor, J.W. and D.O. Natvig. 1987. Isolation of fungal DNA. In Zoosporic fungi in teaching and research (ed. M.S. Fuller and A. Jaworskyl, pp. 252-258. Southeastern Publishing Co., Athens, GA.

Vollmer, S.J. and C. Yanofsky. 1986. Efficient cloning of genes of Neurospora crassa. Proc. Natl. Acad. Sci. 83: 4869-4873.

Wessels, J.G.H., O.M.H. deVries, S.A. Asgeirsdottir, and F.H.J. Schuren. 1991. Hydrophobin genes involved in formation of aerial hyphae and fruit bodies in Schizophyllum. Plant Cell 3: 793-799.

Williams, S.J., R.M. Bradshaw, J.W. Costerton, and A. Forge. 1972. Fine structure of the spore sheath of some Streptomyces species. J. Gen. Microbiol. 72: 240-258.

Zolan, M.E. and P.J. Pukkila. 1986. Inheritance of DNA methylation in Coprinus cinereus. Mol. Cell. Biol. 6: 195-200. 


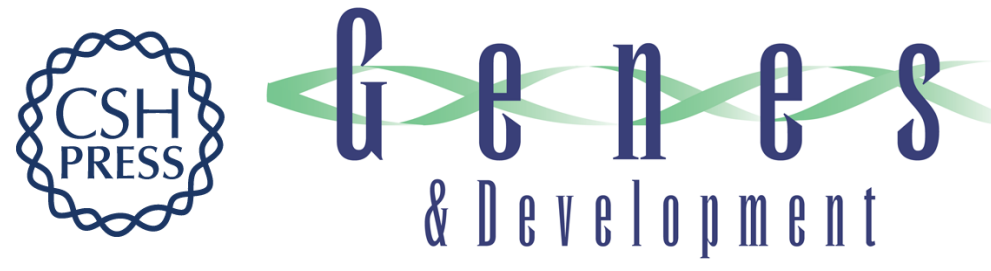

\section{The Neurospora circadian clock-controlled gene, ccg-2, is allelic to eas and encodes a fungal hydrophobin required for formation of the conidial rodlet layer.}

D Bell-Pedersen, J C Dunlap and J J Loros

Genes Dev. 1992, 6:

Access the most recent version at doi:10.1101/gad.6.12a.2382

References This article cites 46 articles, 19 of which can be accessed free at:

http://genesdev.cshlp.org/content/6/12a/2382.full.html\#ref-list-1

License

Email Alerting Service

Receive free email alerts when new articles cite this article - sign up in the box at the top right corner of the article or click here.

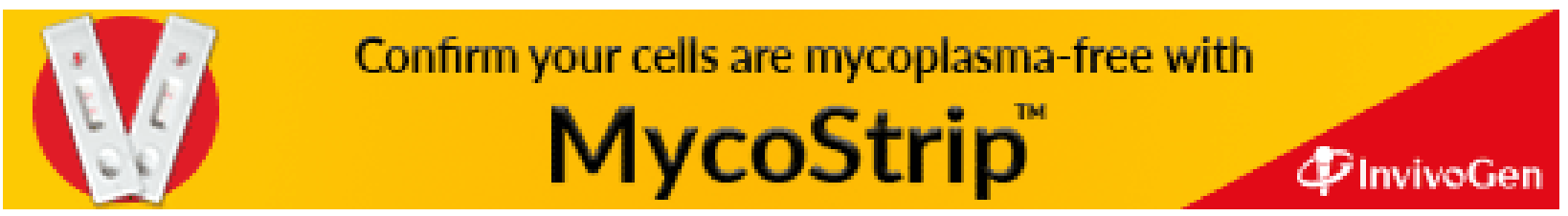

